\title{
College submission to the Department of Health High Security Working Group: a summary
}

\section{Background}

After completion of the review of Health \& Social Services for Mentally Disordered Offenders, and others requiring similar services (the Reed Committee), Dr John Reed took on the new task of reviewing high security services. His working group, which was established in late 1992 , has now reported to Ministers, but the recommendations have not yet been published.

In February 1993 the Forensic Executive Committee prepared an advisory paper which was approved by the College and presented to the Reed High Security Group. This paper is summarised below.

\section{Is high security hospital treatment necessary?}

It is acknowledged that there would always be certain patients who could only be managed under conditions of high security. The size of this patient group is unclear, but a 'best guess' estimate was of around 1,000 patients annually in England and Wales. It was recommended that services for this patient group should shift from the three large special hospitals towards five or six (200-250 bed units) to meet the principle proposed by the original Reed Committee that patients should be managed as near as possible to their own homes or families. These smaller high security units would also be able to develop closer working relationships with local general and forensic psychiatry services. Such changes would encourage the unimpeded flow of patients through the various levels of security so that another of the 'Reed guiding principles' could be met - that patients should be treated and cared for under conditions of no greater security that is justified by the degree of danger they represent to others. It was suggested that in some cases these smaller high security units could be managed by the same provider service responsible for the local medium secure unit.

\section{Do clinical practice arrangements need to change?}

Clinical services in high security hospitals must have the same autonomy to make clinical decisions as would be acceptable elsewhere in the health service. Everything should be done to ensure that adequate numbers of quality staff are employed. To achieve this goal for psychiatry, consultants should be responsible for no more than 45 in-patients, and they should have some sessional commitments outside the hospital. Medical staff should also be encouraged to whistle blow' and to approach management direct if standards of care and treatment are thought to be unacceptable. It was also suggested that professional advice to C3 Division in the Home Office on restricted patients could be organised by the latter seeking sessional input from 'experienced' psychiatrists and other relevant professionals. No consensus exists with regard to psychopathically disordered patients.

\section{A national inspectorate?}

Bearing in mind the failure of the Mental Health Act Commission, other visiting bodies, and the membership of our College to identify and remedy serious deficiencies at Ashworth Hospital (as highlighted in the recent Inquiry into Complaints about Ashworth Hospital) a new Inspectorate was recommended. This Inspectorate could report directly to the Secretary of State for Health and its remit could extend beyond high security hospitals to include medium secure units and perhaps even health care centres in prison establishments - all facilities that struggle each day with the ethical dilemmas and practical problems arising from psychiatric treatment under conditions of security.

Finally it was proposed that the Special Hospital Service Authority (SHSA) should not continue as a central agency with a provider management function, or to have a longterm commissioning role. It was suggested that there is an urgent need to clarify the role of the SHSA and its changing functions over the short, medium and long-term.

Peter Snowden and David Finnegan, Forensic Section, The Royal College of Psychiatrists 